\title{
Original Article \\ Markers of Poor Prognosis in Non-ST Segment Elevation Acute Coronary Syndromes without Revascularization: A 3-year Survival Analysis
}

\author{
Alexander Parkhomenko, Natalia Dovgan, Yaroslav Lutay, Sergey Kozhukhov \\ Department of Emergency Cardiology, NSC Institute of Cardiology by M. D. Strazhesko, Kyiv, Ukraine \\ Address for correspondence: Sergey Kozhukhov, Department of Emergency Cardiology, NSC Institute \\ of Cardiology by M. D. Strazhesko, Kyiv, Ukraine. E-mail: s.kozhukhov@i.ua
}

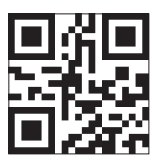

Quick Access Code

How to cite this article:

Parkhomenko A,

Dovgan N, Lutay Y, Kozhukhov S. Markers of Poor Prognosis in NonST Segment Elevation Acute Coronary Syndromes Without Revascularization: A 3-year Survival Analysis. Journal of Medical Research and Innovation. 2018;2(2):e000139.

Doi: 10.15419/jmri.139

Publication history: Received: 09-06-2018 Accepted: 16-07-2018 Published: 17-07-2018

Editors: Dr. Varshil Mehta

Copyright: Parkhomenko A. This is an open access article distributed under the terms of the Creative Commons Attribution License CC-BY 4.0., which permits unrestricted use, distribution, and reproduction in any medium, provided the original author and source are credited.

Funding: NIL Conflict of Interest: NIL

\section{Abstract}

Introduction: The non-ST elevation-acute coronary syndrome (NSTE-ACS) accounts for more than $50 \%$ of the total number of patients with ACS. The mortality rates after non-ST-elevation myocardial infarction (MI) are not significantly different when compared with patients with ST-segment elevation MI. The aim of the present study was to investigate whether the assessment of clinical, laboratory, and instrumental data during hospital stay provides any additional independent information in predicting the 3-year major cardiac events after NSTE-ACS. Patients and Methods: We observed 490 consecutive patients, who were admitted to the emergency cardiology department with NSTE-ACS. The patients' baseline characteristics, blood analysis, left ventricle (LV), and renal function data were assessed and analyzed. The median followup time was 36 months. The endpoint was cardiovascular death. Results: The results of our study show that the risk of cardiovascular death during the 3 years' followup after multivariate adjustment increases with older age ( $>64$ years), history of diabetes, prior $\mathrm{MI}$ and history of angina pectoris, lower ejection fraction $(<50 \%)$, degree of myocardial hypertrophy (the thickness of the interventricular septum $>1.25 \mathrm{~mm}$ ) of the LV and the degree of diastolic dysfunction (E-wave deceleration time $<150 \mathrm{~ms}$ ), silent myocardial ischemia during first 24-h, high pulse pressure on day 1 (>49 $\mathrm{mm} \mathrm{Hg}$ ), glucose level $>7.5 \mathrm{mmol} / \mathrm{l}$ on admission, and moderate kidney dysfunction (creatinine level and its clearance $<60 \mathrm{ml} / \mathrm{min}$ ). Conclusion: In patients with NSTE-ACS, we report the cardiovascular death risk factors within the 3-year follow-up period in the present study. We, thus, conclude that it is important to identify the patients with high risk of future cardiovascular complications.

Keywords: Myocardial infarction without ST-elevation, unstable angina, marker of prognosis, survival

\section{Introduction}

Coronary artery disease remains one of the leading causes of death worldwide. Approximately 1 million new and recurrent acute myocardial infarctions (AMI) occur annually in the United States. ${ }^{[1]}$ Each year in Ukraine, more than 50,000 patients experience an acute coronary event ${ }^{[2]}$ and more than $50 \%$ of them have a non-ST elevation acute coronary syndrome (NSTE-ACS) with increasing of the proportion of NSTE-ACS events per year. ${ }^{[3]}$

Mortality rates after AMI have decreased over the past decades but differ between ST-segment elevation MI (STEMI) and non-STEMI (NSTEMI) patients. ${ }^{[4]}$ Data from randomized trials have shown that hospitalized patients with NSTEMI have a lower risk of death during the $1^{\text {st }}$ few weeks after $\mathrm{MI}$ whereas are at the higher risk for cardiovascular outcomes over the long-term follow-up than those with STEMI. ${ }^{[5,6]}$

Patients with ACS should undergo risk stratification to predict those who are at high risk for short- and long-term adverse outcomes. Among patients with NSTE-ACS, which includes NSTEMI and unstable angina (UA), risk stratification begins soon after admission to detect patients at high risk during the early 
hospital phase. Subsequent risk stratification is aimed to predict patients being at increased risk after discharge. ${ }^{[7]}$

The aim of the present study was to investigate whether the assessment of clinical, laboratory, and instrumental data during hospital stay provides additional independent information in predicting 3-year major cardiac events after NSTE-ACS.

\section{Patients and Methods}

\section{Study population}

In an observational, retrospective cohort study, a total of 490 consecutive patients admitted to the emergency cardiology department with a diagnosis of NSTE-ACS were included. The definition of NSTE-ACS was based on the following criteria: (a) Prolonged (>20 min) anginal pain at rest; (b) and electrocardiographic (ECG) findings suggestive of ischemia: ST segment depression ( $>1 \mathrm{~mm}$ ) or inversion of the $\mathrm{T}$ wave (>1 mm); and/or (c) positive biomarkers of necrosis (troponins or MB fraction of creatine kinase).

\section{Inclusion criteria}

The following criteria were included in the study:

1. Patients with NSTE-ACS $<24 \mathrm{~h}$ from the last pain at rest.

2. Changes on the ECG:

- $\quad$ ST depression $>1 \mathrm{~mm}$

- Transient ST elevations $>1 \mathrm{~mm}$

- Inversion of the T wave $>2 \mathrm{~mm}$

- Absence of new changes on the ECG in case of history of MI.

3. $<72 \mathrm{~h}$ from the time of destabilization.

\section{Exclusion criteria}

The following criteria were excluded from the study:

1. Valvular heart diseases

2. Chronic heart failure NYHA III-IV

3. Cardiogenic shock

4. Severe liver dysfunction (AST or ALT $>3 \times$ ULN)

5. Kidney dysfunction (creatinine level and its clearance $[\mathrm{CrCl}]<30 \mathrm{ml} / \mathrm{min}$ )

6. Severe infection

7. Malignant tumor or active cancer.

\section{Study protocol}

Each patient underwent a physical examination with measuring of blood pressure (BP), heart rate, body mass index, and followed by standard diagnostic tests (ECG, biomarkers). Furthermore, we analyzed complications during in-hospital period and laboratory data (C-reactive protein, fibrinogen, erythrocyte sedimentation rate [ESR], white blood cells [WBC], and creatinine) on the day 1 and on the day 10. Two-dimensional echocardiography was performed on the day 1 after admission to the hospital and Holter ECG monitoring and heart rate variability (HRV) on the days $1,3,7$, and 10 of in-hospital stay. Renal function was estimated using $\mathrm{CrCl}$ on admission and on the day 10 using the Cockcroft-Gault formula. Treatment of all patients was according to the National and European recommendations and standards. ${ }^{[7]}$

This study was approved by the Ethics Committee at the National Scientific Center, the M. D. Strazhesko Institute of Cardiology.

\section{Endpoints}

The endpoint was a composite of cardiovascular death, non-fatal $\mathrm{MI}, \mathrm{UA}$, and percutaneous coronary intervention with coronary artery stenting and coronary artery bypass grafting. Information regarding adverse events was obtained from patients or family doctors or patients' relatives during phone calls.

\section{Statistical analysis}

Statistical analysis was assessed with "SPSS 13.0" software using Student's t-test and $\chi^{2}$-test. Data were reported as means with standard deviation, and the categorical variables were expressed as numbers with percentages. A multivariate Cox regression analysis was performed for the confounding effects, and odds ratios and $95 \%$ confidence intervals were calculated. A value of $P<0.05$ was considered statistically significant.

\section{Results}

\section{Patients' characteristics}

All prognostic analyses in the present study were carried out using data from 490 patients. The baseline characteristics of the patients and medications are summarized in Tables 1 and 2 respectively.

\section{Endpoint events}

The median follow-up time was 36 months (interquartile range: 32-39 months). The endpoint was monitored every 6 months, and the data of $94.90 \%$ of patients $(n=465)$ were available for analyses. Patients, who died during in-hospital stay and those without contact during follow-up, were excluded from analyses. Endpoint events during 3-year follow-up are presented in Table 3.

Results of our study show that the risk of cardiovascular death during 3-year follow-up after multivariate adjustment increases with older age (>64 years), history of diabetes, prior $\mathrm{MI}$, and history of angina pectoris [Figure 1].

Lower ejection fraction (EF) $(<50 \%)$, degree of myocardial hypertrophy (the thickness of the interventricular septum $>1.25 \mathrm{~mm}$ ) of the left ventricle (LV), and the degree of diastolic dysfunction (E-wave deceleration time [DT] $<150 \mathrm{~ms}$ ) were identified as independent predictors of cardiovascular mortality also [Figure 2]. 
We hypothesized that depressed HRV could have a negative impact on cardiovascular death by suppressing parasympathetic and augmenting sympathetic components of HRV. Highfrequency $(\mathrm{PH}<18.5)$ and low-frequency $(\mathrm{PL}>81)$ components of HRV and total power (PT $>15$ ) were determined by spectral analysis [Figure 3].

Silent myocardial ischemia (SMI)-objectively documented ischemia in the absence of chest discomfort or an another

Table 1: Study baseline characteristics

\begin{tabular}{lc} 
Number & $n=490$ \\
Clinical observation & \\
Age, years & $58,78 \pm 0.44$ \\
Male & $365(74.49 \%)$ \\
Hypertension & $379(77.35 \%)$ \\
Coronary artery disease & $355(72.04 \%)$ \\
DM & $79(16.12 \%)$ \\
Current smoker & $167(34.08 \%)$ \\
History of chronic heart failure & $51(10.41 \%)$ \\
History of MI & $194(39.59 \%)$ \\
BMI >30 kg/m² & $81(16.53 \%)$ \\
ECG & \\
Depression of ST segment & $208(42.45 \%)$ \\
Inverted T wave & $277(56.53 \%)$ \\
Transient ST-segment elevation & $42(8.57 \%)$ \\
\hline
\end{tabular}

DM: Diabetes mellitus, MI: Myocardial infarction, BMI: Body mass index,

ECG: Electrocardiographic

Table 2: Treatment baseline characteristics

$\begin{array}{lc}\text { Medications } & n=490 \\ \text { Antiplatelet } & 490(100 \%) \\ \text { ASA } & 437(89.18 \%) \\ \text { Thienopyridines } & 143(29.18 \%) \\ \text { LMW heparins } & 357(72.86 \%) \\ \text { Standard heparin } & 78(15.92 \%) \\ \text { Beta-blockers } & 404(82.45 \%) \\ \text { ACE inhibitors } & 347(70.82 \%) \\ \text { Nitrates (oral) } & 337(68.78 \%) \\ \text { Nitrates (i.v.) } & 311(63.47 \%) \\ \text { Lipid-lowering } & 203(48.78 \%) \\ \text { therapies } & \end{array}$

Table 3: Endpoint events during 3-year follow-up

\begin{tabular}{lc} 
Endpoint events & $\boldsymbol{n}=465$ \\
Cardiovascular death & $58(12.47 \%)$ \\
Non-fatal MI & $38(8.17 \%)$ \\
UA & $81(17.42 \%)$ \\
PTCA with stenting & $56(12.04 \%)$ \\
CABG & $55(11.83 \%)$ \\
\hline
\end{tabular}

UA: Unstable angina, MI: Myocardial infarction anginal equivalent - is a major risk factor of poor prognosis. ${ }^{[8]}$ We studied SMI during days 1, 3, 7, and 10 by $24 \mathrm{~h}$ Holter ECG monitoring, and only SMI during the first $24 \mathrm{~h}$ had a negative predictive impact.

Risk of Cardiovascular Death

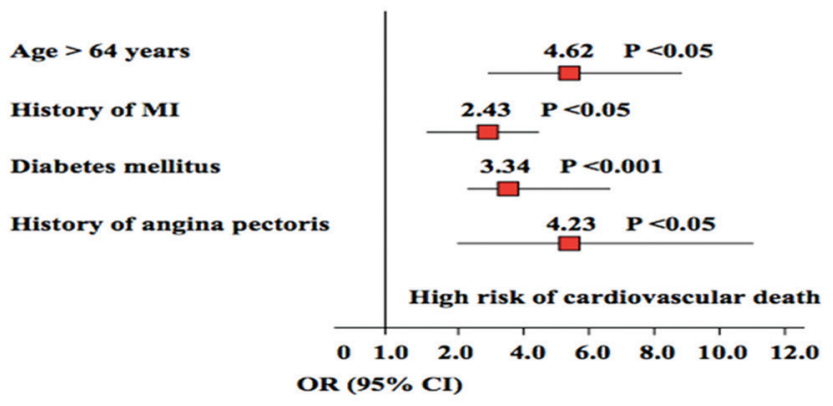

Figure 1: Impact of age, history of myocardial infarction, diabetes mellitus, and angina pectoris on cardiovascular death

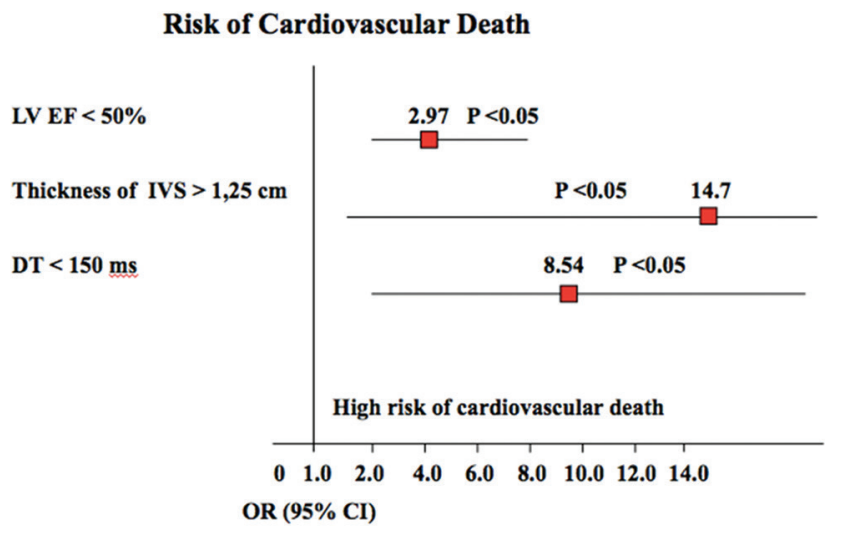

Figure 2: Impact of left ventricle ejection fraction, myocardial hypertrophy, and diastolic dysfunction on cardiovascular death

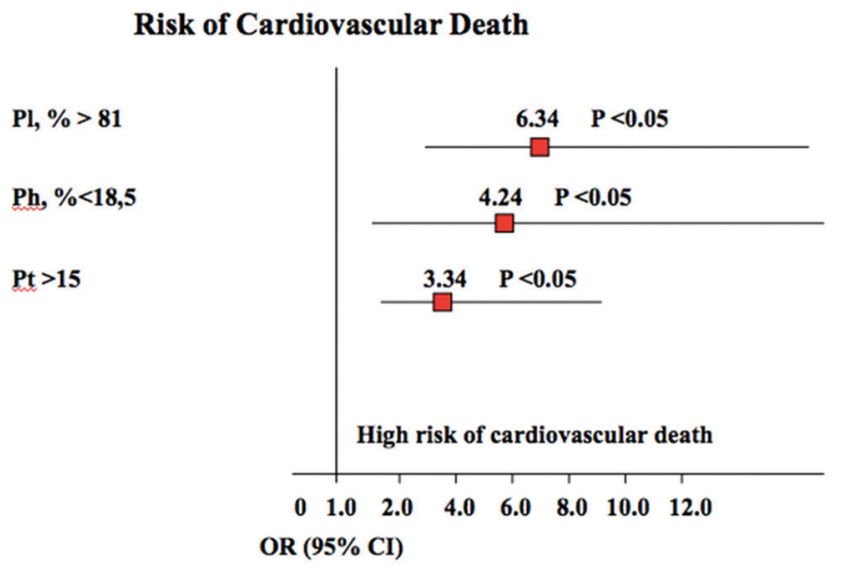

Figure 3: Impact of heart rate variability on cardiovascular death 
Clinical history of arterial hypertension in $\mathrm{MI}$ is well known as a negative prognostic factor. ${ }^{[9]}$ In our study, we established that low mean and high pulse pressure on the day 1 were significantly associated with cardiovascular mortality [Figure 4].

The high ESR levels on admission are found to be related to poor short- and long-term survival. Therefore, ESR evaluation on admission may be helpful to identify patients with a poor prognosis. In our study value, ESR $>13 \mathrm{~mm} / \mathrm{h}$ on admission was a cutoff point according to the Cox regression analyses.

Patients either with or without a prior history of diabetes mellitus (DM) may present with hyperglycemia during ACS, and hyperglycemia on admission remains an independent predictor of post-discharge mortality. Our data confirmed that glucose level $>7.5 \mathrm{mmol} / \mathrm{l}$ on admission could be a negative risk factor for those patients without a history of DM.

Renal function should be measured in all patients with NSTEACS during $1^{\text {st }} \mathrm{h}$ because it is important for dose adjustment of drugs and contrast agents released through the kidneys. On the other hand, estimated glomerular filtration rate (GFR) has a powerful relationship between the severity of renal dysfunction and poor outcomes. In our study, survival analysis showed that patients with moderate dysfunction $(\mathrm{CrCl}<60 \mathrm{ml} / \mathrm{min})$ had a higher rate of cardiovascular death than patients with $\mathrm{CfCl}$ $>60 \mathrm{ml} / \mathrm{min}$ [Figure 5].

\section{Discussion}

Optimal, evidence-based treatment after NSTE-ACS can only reduce the risk of an event. Even optimally treated patients face a residual risk of adverse cardiovascular outcomes due to the underlying disease process and a number of comorbidities. ${ }^{[10]}$ Therefore, all post-NSTE-ACS patients should receive betablockers and statins in recommended doses permanently, if not contraindicated. ${ }^{[11]}$ Patients also require medications to modify risk factors, such as antihypertensive medications to achieve target $\mathrm{BP}$, angiotensin-converting enzyme inhibitors for left ventricular dysfunction, and antihyperglycemic agents to maintain a goal of glucose level. ${ }^{[12]}$

In our study, we investigated the risk factors for the stratification of long-term outcomes. It is very important to identified patients with a high risk of future cardiovascular complications after discharge. The results of this study showed that older age, history of $\mathrm{MI}$, previously diagnosed angina pectoris, diabetes, systolic dysfunction, HRV abnormality, elevated mean pulse BP and blood glucose findings at the time of hospital admission, and lower $\mathrm{CrCl}$ findings were the main factors significantly associated with an adverse long-term prognosis following hospital discharge for patients with NSTE-ACS after multivariate adjustment for the demographic characteristics, comorbidities, and hospital complications.

\section{Risk of Cardiovascular Death}

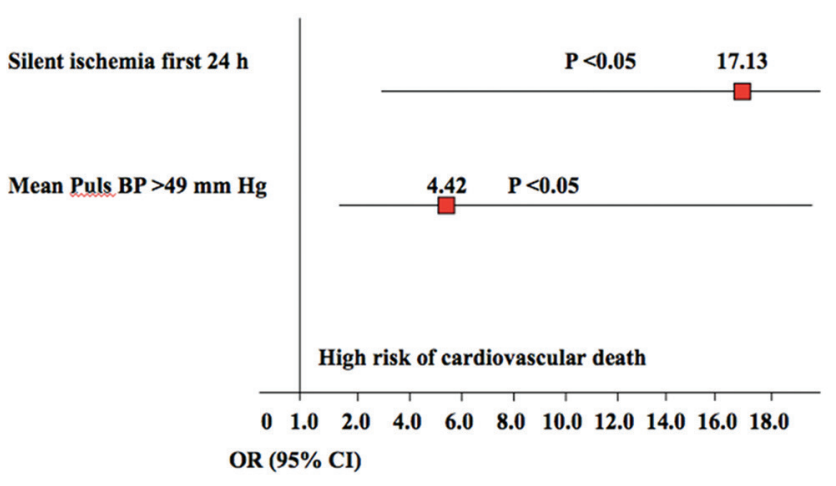

Figure 4: Impact of silent myocardial ischemia and mean pulse blood pressure on cardiovascular death

\section{Risk of Cardiovascular Death}

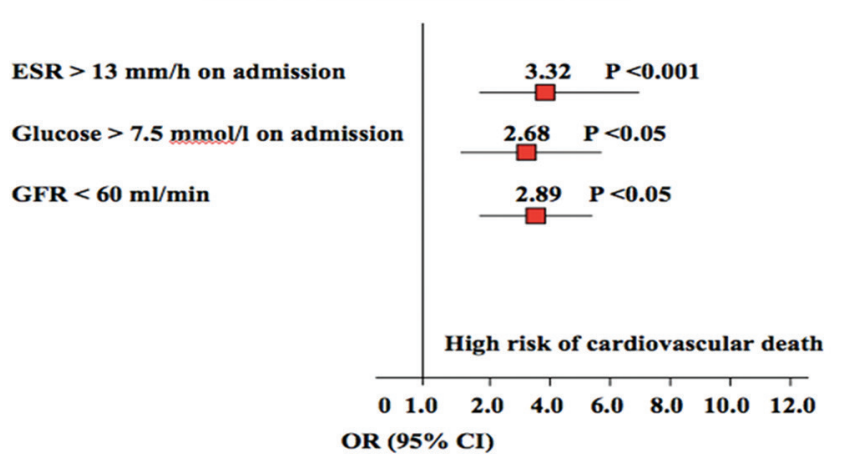

Figure 5: Impact of erythrocyte sedimentation rate, serum glucose, and glomerular filtration rate on cardiovascular death

Older age and the presence of comorbidities have been recognized as critical factors in predicting the clinical outcomes in patients with ACS. In the present study, it was proved that existing coronary heart disease, DM, and renal disease were the main comorbidities led to a higher incidence of mortality. In an another large study, it was found that independent predictors of early mortality in NSTE-ACS patients included old age above 60-year-old, Killip class, female gender, LV dysfunction, and renal failure. ${ }^{[12]}$ In another such study, it was confirmed that DM, in addition to age and systolic LV dysfunction, was found to be independent predictors of early mortality in those patients. ${ }^{[13]}$

There are studies that suggest that hemodynamic measures can predict mortality, whereas the evidence of the prognostic value of hemodynamic measures in NSTE-ACS patients is limited. This research demonstrated the influence of LV hypertrophy and the degree of diastolic dysfunction on CV death, and our data coincide with the results of other authors. ${ }^{[14]}$

LVEF is a valid indicator of myocardial function and has shown by multiple studies to be an established predictor of adverse 
clinical outcomes. Data of a prospective cohort study showed that LVEF $\leq 50 \%$ was associated with high rates of adverse events, including death. ${ }^{[15]}$

Low HRV is usually considered as a negative long-term prognostic factor after an ACS. In our group of patients with a recent ACS, abnormal autonomic parameters have been correlated with long-term outcomes. These results support the significant prognostic value of traditional HRV parameters, and HRV measured close to the ACS onset may assist in risk stratification. ${ }^{[16]}$

According to the recent publications, the long-term mortality for patients with NSTE-ACS has been related to the extent of their comorbid disease burden. ${ }^{[17]}$ In the present study, patients with NSTE-ACS were more likely to have several previous comorbid diseases, especially patients to have decreased survival if they had a history of angina pectoris, prior $\mathrm{MI}$, and diabetes. Similarly, we found that systolic dysfunction and heart rate abnormalities were associated with decreased survival after hospital discharge.

If established risk assessment methods are not used, it leads to underestimate risk in high-risk patients and overestimate risk in low-risk patients. ${ }^{[18]}$ In addition, physicians tend to estimate the risk based on the intensity of treatment received during the ACS. In particular, physicians underestimate risk associated with age and may consider younger ACS patients as having a more aggressive disease phenotype than older patients, while underestimating the impact of age-associated accumulated coronary artery damage. ${ }^{[19]}$ Therefore, it is important that physicians should use validated objective measures of risk when assessing ACS patients.

\section{Limitations}

Our study has several limitations that should be considered when interpreting the present results. This was a single-center and observational uncontrolled study. The samples were restricted to patients discharged from our hospital with a successful follow-up, which may have resulted in selection biases and conclusions with limited generalizability. There was a tendency for cardiologists to perform conservative treatment for high-risk patients, which might have affected the final results. Finally, this study cannot exclude possible residual confounding by other measured and/or unmeasured factors including the treatment decisions of patients when NSTE-ACS occurred, which is an important source of prognosis.

There are currently limited data to guide clinical decisionmaking around optimal secondary preventive therapies in NSTEACS patients who survive after hospital discharge. On-going risk assessment is important in all post-MI patients, and clinicians should use the objective measures of evaluation whenever it possible, to avoid over- or under-estimating future risk.

\section{Conclusion}

Our findings suggested that, in patients with NSTE-ACS, the cardiovascular death risk factors for the 3-year follow-up were as follows:

- Demographic and clinical factors: Age, history of $\mathrm{MI}$, history of angina pectoris, and DM.

- Instrumental: LV EF, LV hypertrophy, low DT, depressed $\mathrm{HRV}$, and silent ischemia according to the results of Holter ECG monitoring and median pulse BP.

- Laboratory: ESR, glucose level, and GFR.

Future research efforts should focus on the study risk factors in patients after NSTE-ACS and evaluate the impact on long-term outcomes.

\section{References}

1. Benjamin EJ, Blaha MJ, Chiuve SE. Heart disease and stroke statistics-2017 update: A report from the American heart association. Circulation 2017; 135:e146-603.

2. Number of Deaths by Sex, Age Groups and Causes of Death. Departament Statystyky Naselennia ta Rehional'noyi Statystyky. Derzhkomstat Ukrayiny. Available from: http:// www.ukrstat.gov.ua/druk/publicat/kat_u/2017/bl/06/ bl_zoz_2016.zip. [Last accessed on 2018 Jun 06].

3. Terkelsen CJ, Lassen JF, Nørgaard BL, Gerdes JC, Jensen T, Gøtzsche LB. Mortality rates in patients with ST-elevation vs. non-ST-elevation acute myocardial infarction: Observations from an unselected cohort. Eur Heart J 2005;26:18-26.

4. Montalescot G, Dallongeville J, Van Belle E. OPERA investigators STEMI and NSTEMI: Are they so different? 1 year outcomes in acute myocardial infarction as defined by the ESC/ACC definition (the OPERA registry). Eur Heart J 2007;28:1409-17.

5. Cantor WJ, Goodman SG, Cannon CP. Early cardiac catheterization is associated with lower mortality only among high-risk patients with ST- and non-ST-elevation acute coronary syndromes: Observations from the OPUSTIMI 16 trial. Am Heart J 2005;149:275-83.

6. Cox DA, Stone GW, Grines CL. CADILLAC investigators comparative early and late outcomes after primary percutaneous coronary intervention in ST-segment elevation and non-ST-segment elevation acute myocardial infarction (from the CADILLAC trial). Am J Cardiol 2006;98:331-7.

7. Roffi M, Patrono C, Collet JP, Mueller C, Valgimigli $M$, Andreotti F, et al. 2015 ESC Guidelines for the management of acute coronary syndromes in patients presenting without persistent ST-segment elevation: Task force for the management of acute coronary syndromes in patients presenting without persistent ST-segment elevation of the European society of cardiology. Eur Heart J 
2015;37:267- 315.

8. Pelter MM, Loranger DL, Kozik TM. Among unstable angina and non-ST rlevation MI patients, transient myocardial ischemia and early invasive treatment are predictors of major in-hospital complications. J Cardiovasc Nurs 2016;31:E10-9.

9. Rembek M, Goch A, Goch J. The clinical course of acute ST-elevation myocardial infarction in patients with hypertension. Kardiol Pol 2010;68:157-63.

10. Savonitto S, Cavallini C, Petronio AS, Murena E, Antonicelli R, Sacco A. Early aggressive versus initially conservative treatment in elderly patients with nonST-segment elevation acute coronary syndrome: A randomized controlled trial. JACC Cardiovasc Intervent 2012;5:906-16.

11. Smith SC Jr, Benjamin EJ, Bonow RO. World Heart Federation and the Preventive Cardiovascular Nurses Association. AHA/ACCF secondary prevention and risk reduction therapy for patients with coronary and other atherosclerotic vascular disease: 2011 update: A guideline from the American Heart Association and American College of Cardiology Foundation. Circulation 2011;124:2458-73.

12. Park H, Yoon C, Kang S. Early- and late-term clinical outcome and their predictors in patients with ST-segment elevation myocardial infarction and non-ST-segment elevation myocardial infarction. Int J Cardiol 2013;169:254-61.

13. Savonitto S, Cavallini C, Petronio AS, Murena E, Antonicelli R, Sacco A. Early aggressive versus initially conservative treatment in elderly patients with nonST-segment elevation acute coronary syndrome: A randomized controlled trial. JACC Cardiovasc Interv 2012;5:906-16.

14. Stork T, Mockel M, Danne O, Voller H, Eichstadt H, Frei U. Left ventricular hypertrophy and diastolic dysfunction: Their relation to coronary heart disease. Cardiovasc Drugs Ther 1995;9 Suppl 3:533-7.

15. Vakili H, Sadeghi R, Rezapoor P, Gachkar L. In-hospital outcomes after primary percutaneous coronary intervention according to left ventricular ejection fraction. ARYA Atheroscler 2014;10:211-7.

16. Harris $P$, Stein $P$, Fung $G$, Drew $B$. Heart rate variability measured early in patients with evolving acute coronary syndrome and 1-year outcomes of rehospitalization and mortality. Vascular Health Risk Manage 2014;10:451-64.

17. Worrall-Carter L, McEvedy S, Wilson A, Rahman M. Impact of comorbidities and gender on the use of coronary interventions in patients with high-risk non-ST-segment elevation acute coronary syndrome. Catheter Cardiovasc Interv 2016;87:E128-36.

18. Chew DP, Junbo G, Parsonage W. Perceived risk of ischemic and bleeding events in acute coronary syndromes. Circ Cardiovasc Qual Outcomes 2013;6:299-308.

19. Yan AT, Yan RT, Huynh T. Understanding physicians' risk stratification of acute coronary syndromes: Insights from the Canadian ACS 2 Registry. Arch Intern Med 2009;169:3728. 